Title:

\title{
Adolescent Sexual Wellbeing: A Conceptual Framework
}

\author{
Anna Kågesten ${ }^{1 *}$ and Miranda van Reeuwijk ${ }^{2}$
}

1. Department of Global Public Health, Karolinska Institutet, Tomtebodavägen 18A, 17177 Stockholm, Sweden

2. Rutgers. Arthur van Schendelstraat 696, 3511 MJ Utrecht, The Netherlands

*Corresponding author: Anna Kågesten, Department of Global Public Health, Tomtebodavägen 18A, 17177 Stockholm, Sweden. Email: anna.kagesten@ki.se

\begin{abstract}
Positive aspects of sexuality remain understudied among adolescents globally, especially in low- and middle-income countries. In particular, consensus is lacking on how to conceptualize different aspects of sexual wellbeing among young people. We propose a new conceptual framework for adolescent sexual wellbeing that draws on existing theories and definitions, grounded in theories related to positive youth development, empowerment, human rights, gender, social-ecological and life-course perspectives. The framework highlights six interrelated domains of key competencies that are central to healthy adolescent sexuality development, whether or not sexual activities occur: 1) sexual literacy, 2) gender equal attitudes, 3) respect for human rights and understanding consent, 4) critical reflection skills, 5) coping skills and 6) interpersonal skills. These competencies have the potential both to strengthen and impede adolescents' sexual wellbeing in relation to both themselves and others. Personal sexual wellbeing includes, but is not limited to, having a positive sense of one's own (sexual) self and body, and recognising and appreciating feelings of pleasure and desire. Relational sexual wellbeing can include developing and maintaining mutually respectful, gender equal relationships with other people that are that are part of young people's sexual socialisation, such as peers, parents and community members. Over time, relational sexual wellbeing may also include outcomes related to intimate partners, such as equality in relationships, safe and pleasurable sexual interactions, free from violence and coercion. Whether young people are able to translate competencies into desired actions and achieve a sense of sexual wellbeing depends on the personal, social and economic resources available to them, their (sexual) agency, and on the influence of opportunity structures such as policies, laws and norms. We end with discussing the potential implications of the framework for public health programmes and research studies aiming to improve adolescent SRHR and wellbeing.
\end{abstract}

\section{Word count:}

Abstract: 293

Article: 3880

Number of references: 40 
A growing body of scholars and stakeholders are calling for "positive" approaches to adolescent sexuality, ${ }^{1-7}$ moving beyond the predominant focus of research and programs on preventing risky outcomes such as unintended pregnancies and HIV. ${ }^{8}$ While the latter is not strange from a public health perspective (certainly, it is a lot easier to justify studying a sensitive topic because of its potential risks, as opposed to focusing on any positive aspects which may be both taboo and difficult to measure $)^{2}$, such a risk-based approach is problematic in a number of ways.

First, it assumes that adolescent sexuality and related behaviors are a priori problematic, while in fact they are part of a normative developmental process which begins at (or even before) birth. ${ }^{7}$ The onset of puberty triggers a cascade of physical, cognitive, emotional and social changes, with young people gradually starting to explore their sexual identity, to form intimate relationships, and to experiment with different practices. ${ }^{9}$ Second, risk and dangeroriented messages and interventions including abstinence-only programs have been shown not to be effective to prevent sexual risk; and to clash with adolescent's needs, realities and human rights to explore their sexuality, experience pleasure, and to seek and receive comprehensive information and services. ${ }^{6,10}$ And finally, because sexual activity is almost always conceptualized in terms of penile-vaginal intercourse, and rarely in terms of relationships, pleasure, feelings and other sexual practices, programs tend to focus on older, heterosexual youth - thereby overlooking what is needed to promote sexual wellbeing among a diversity of adolescents, including sexual minorities and younger age-groups. ${ }^{3}$

Moving from a risk to a positive perspective on adolescent sexuality does not mean that all young people are (or should be) having sex, or that all intimate relations are healthy. It merely implies that sexual wellbeing is more than (just) avoiding risks and unwanted consequences. ${ }^{3,4}$ However, despite the strive to shift towards positive approaches, consensus is lacking around how to define and measure broader, affirmative aspects of sexual wellbeing in order to inform research and interventions. ${ }^{5,11}$ What exactly does positive or healthy sexuality development entail during adolescence, including the earliest years (ages 10-14) which is a critical period for shaping attitudes and behaviors? ${ }^{9}$

In this paper, we propose a conceptual framework for adolescent sexual wellbeing with the goal of offering guidance to research and programs aiming to improve sexual and reproductive health and rights (SRHR). We begin with a background on adolescent sexuality development and wellbeing based on existing definitions and conceptualizations. We then move on to explain the different components of the framework and end with discussing its potential implications for public health professionals.

\section{Background}

\section{Adolescent sexuality as a developmental process}

As a normative process, adolescent sexuality development does not occur in isolation, but interacts with other developmental facets (e.g. the formation of morality, values, identity), and is shaped by the complex interplay between individuals and their social-ecological environment. ${ }^{2,3,7}$ Such a perspective is rooted in Developmental Systems Theory and Positive Youth Development (PYD), viewing young people as resources to be nurtured rather than problems to be fixed. ${ }^{12,13}$ Evidence shows that building on adolescents' developmental assets in line with PYD can have both immediate and long-term positive effects on their health and wellbeing. ${ }^{14}$ For example, Blum et al. ${ }^{9}$ propose four central outcomes of healthy development during early adolescence, including: life and decision-making skills, self-efficacy, emotional and physical safety, and engagement in learning; each of which have been linked with sexual and reproductive health later in adolescence. Further, a 2016 review found that positive aspects 
of sexuality is linked not only with sexual health, but with physical health, mental health as well overall wellbeing. ${ }^{15}$

As noted by Halpern" " "a fine line may divide exploratory sexual activity that ultimately contributes to positive sexual identity and competence, and sexual activity that significantly increases risk of harm". From a developmental system and PYD perspective, the role of public health programs is to help young people navigate that "fine line" which may divide potentially positive sexual experiences from those that are harmful. ${ }^{2}$ In order to do so, research and interventions must include meaningful efforts to understand and strengthen young people's assets, ultimately affecting their wellbeing. Below we take a closer look at existing models that have attempted to conceptualize sexual wellbeing and sexuality from such an affirmative perspective.

\section{Defining “healthy" adolescent sexuality and wellbeing}

The World Health Organization (WHO) defines sexual health as a "state of physical, emotional, mental and social wellbeing in relation to sexuality", including the possibility of experiencing "pleasurable and safe sexual experiences" and emphasizing individuals' sexual rights, such as choosing if, when and with whom to be sexually active, marry, and have children. ${ }^{16}$ This positive, rights-based view on sexuality is further underscored in the recent Guttmacher-Lancet Commission integrated definition of sexual and reproductive health and rights (SRHR), which highlights the role of trust and communication in forming pleasurable sexual relationships, and in promoting self-esteem and overall wellbeing. ${ }^{17}$ Similarly, over two decades ago the US National Commission on Adolescent Health defined sexual health as the "ability to develop and maintain meaningful interpersonal relationships; appreciate one's own body; interact with both genders in respectful ways; and express affection, love and intimacy in ways consistent with one's own values." 18

A number of scholars have further attempted to conceptualize adolescent sexuality from a positive perspective. ${ }^{1,3,6,19}$ For example, Harden ${ }^{3}$ defines adolescent sexual wellbeing as spanning four key dimensions: sexual self-esteem; sexual self-efficacy; feelings of arousal, satisfaction, and pleasure; and freedom from pain, anxiety, and negative effects regarding sexuality. Not engaging in sex (abstinence) could thus reflect a high sense of wellbeing in the form of an active choice, or low levels of wellbeing (for example via low sexual self-esteem or ability to communicate feelings to a partner). ${ }^{3}$ McKee et al. ${ }^{19}$ describe healthy adolescent sexual development as young people having a basic understanding (e.g., of the body) as well as positive attitudes and skills to support sexuality development. They further emphasize agency and resilience as key dimensions, and that a life-course perspective is central where children are "naturally curious about their bodies and sexualities". Arbeit ${ }^{1}$ focus more specifically on skills for positive adolescent sexuality development, including: sexual selfhood (e.g. desire, ethics), sexual negotiation with others (e.g. consent, pleasure), and sexual empowerment (e.g. boundaries), which in turn are linked via personal intimacy, agency and social advocacy. She points to how an individual's sexual selfhood may shape their agency to negotiate with others, thereby affecting intimacy and leading to empowerment.

These frameworks have in common that they emphasize healthy sexuality development as encompassing knowledge, skills and attitudes, which in turn affect individual's sense of sexual wellbeing in relation to themselves as well as others. Indeed, a recent review found that operational definitions of sexual wellbeing span both individual and interpersonal/relational as well as sociocultural dimensions. It emphasized the need to conceptualize sexual wellbeing as "individually experienced, but socially and structurally influenced", and to apply a capability lens that focuses on what young people are "able to be and do" rather than what they "have and do". ${ }^{11}$ In essence, this means that sexual wellbeing is about sexual empowerment, where agency 
and resources play a key role in determining adolescents' ability to navigate their surrounding contexts.

\section{Empowerment as central to sexual wellbeing}

Sexual empowerment can be thought of as the processes of expanding young people's choice and strengthening their voice related to sexuality through the transformation of power relations, whereby they are able to assert their opinions, desires and interests, influence and make purposeful decisions, and challenge existing norms and structures. ${ }^{20,21}$

According to Kabeer, ${ }^{22}$ three interlinked dimensions are central to (sexual) empowerment: agency, resources and achievements. Agency refers to the ability to make and influence decisions (choice), assert own interests and opinions (voice) - both of which are affected by power. Agency is closely connected to the resources or assets available to a person (ref), including both those proximate (such as social capital) as well as surrounding opportunity structures including social norms, laws and policies. ${ }^{21,22}$ For example, Kwauk and Braga ${ }^{23}$ describe how knowledge (what one knows), attitudes (what one believes and values) and skills (what one has) form a broader set of resources or "competencies" (what one can do), ultimately affecting young people's ability to achieve health outcomes. Whether or not a young person is able to translate competencies into empowered action (towards desires outcomes) depends both on their own agency and the agency of others, as well as the degree to which their social context enables change (via opportunity structures).

\section{A conceptual framework for adolescent sexual wellbeing}

Figure 1 presents a conceptual framework for adolescent sexual wellbeing that is intended to guide SRHR research and programs in adolescence and beyond. The framework is based on an extensive narrative review of the literature to identify key components, followed by input from over 20 global experts in the field of adolescent SRHR during workshops, conferences and webinars.

Drawing on the literature, we differentiate between healthy sexuality development as the process through which young people gain knowledge, skills and attitudes that support their own identity formation and navigation of relationships; and sexual wellbeing as the physical, mental, emotional and social/relational wellbeing outcomes related to sexuality - whether or not sexual activity occurs. Specifically, we propose six interrelated domains of key competencies in the form of knowledge, skills and attitudes that are central to healthy adolescent sexuality development. Whether young people are able to translate competencies into desired actions and achieve a sense of sexual wellbeing across the life-course in turn depends on the personal, social and economic resources or assets available to them; their (sexual) agency; and on the influence of social-ecological opportunity structures.

\section{Underlying principles}

The framework is guided by five key principles:

1. First, multiple, interlinked social-ecological factors ${ }^{9}$ shape adolescent sexual wellbeing, including individual, family, school, health systems and wider societal levels.

2. Second, a life-course perspective is implicit in the framework, with adolescence being a key transition point of developmental changes. ${ }^{24}$

3. Third, it draws on a human rights-based, gender transformative approach to recognize adolescents' fundamental right to sexual and reproductive health information, services, participation and non-discrimination. ${ }^{17,25,26}$ It recognizes gender equality as central to adolescent health and wellbeing - in line with recent evidence that gender 
transformative programs can successfully impact health-related knowledge, attitudes and behaviors. ${ }^{27}$

4. Fourth, it uses an empowerment-based approach ${ }^{20-22}$ and capability lens ${ }^{11}$ to highlight how assets (in the form of competencies) interact with agency and opportunity structures to affect sexual wellbeing.

5. Finally, it emphasizes the reciprocal and complex nature of sexuality development, ${ }^{1,3,28}$ for example by pointing to the interconnection between different competencies, and that past experiences inform new ones. For this reason, it is important not to view the different components of the framework as a checklist that one has to achieve, i.e., failure to achieve certain outcomes does not mean that one is "unhealthy".

\section{Sexual wellbeing: personal and relational dimensions}

At the heart of the framework is sexual wellbeing (Figure 1), which we conceptualize as physical, emotional, cognitive and social aspects of sexuality and relations with oneself and others - including both personal and relational dimensions of wellbeing.

By personal sexual wellbeing, we mean aspects such as having a positive sense of one's own (sexual) self, identity and body. It refers to the internal development of the individual, both in terms of the self (e.g. identity, self-esteem, self-efficacy, motivations, feelings and desires) and body (e.g. perceived body image, comfort with puberty changes). ${ }^{1,3,11}$ Developing a positive sense of self is closely linked to the concept of "sexual selfhood" and can include the capacity to be aware of one's sexual desires, ${ }^{1,3,7,19}$ recognizing that it is ok to enjoy these feelings and to experience pleasure, ${ }^{19}$, sexual self-esteem (sense of self-worth and attractiveness) and self-efficacy (perceived ability to assert preferences). ${ }^{3}$

By relational sexual wellbeing, we refer to mutually respectful relationships that are characterized by gender equality and free from violence and coercion. For adolescents and especially the youngest age groups, such relationships do not only refer to intimate partners, but to others in their social contexts such as parents and peers. ${ }^{7}$ Outcomes measuring wellbeing in relation peers might, for example, look at experiences and perpetration of bullying and violence. Over time, relational wellbeing can extend into those related to intimate partners, such as perceived power and communication in intimate relationships, freedom from partner violence, contraceptive use, and whether sexual activities are wanted and pleasurable. ${ }^{1,3,6,7,11,19}$

\section{Key competencies to support healthy sexuality development}

We further propose six key domains of competencies in the form of knowledge, skills and attitudes that support adolescent sexual wellbeing: 1) sexual literacy, 2) gender equitable attitudes, 3) respect for human rights and understanding of consent, 4) critical reflection skills, 5) coping skills and stress management, and 6) interpersonal relationship skills. These competencies can be thought of as internal resources to support healthy sexuality development irrespective of whether a young person has engaged in any sexual activities. Similar to Arbeit's skills-based model, ${ }^{1}$ they form the foundation for how adolescents navigate and explore sexuality in relation to themselves and others.

Sexual literacy refers to an age- and developmentally basic understanding of SRHR. In early adolescence, this might include an understanding of the human body, puberty, emerging feelings and emotions, the meaning of different relationships (e.g. family, friendships, romantic). Whereas, in later adolescence it might expand to knowledge around sexual behavior, SRHR and where to seek services and support. The specific SRHR needs and concerns of adolescents vary within and across regions, countries and communities, and this will affect what is considered both appropriate and relevant information. ${ }^{29}$ 
Gender equitable attitudes points to the importance of holding views that support gender equality. As young people transition into adolescence, they are increasingly exposed to social and cultural expectations and messages about the roles, responsibilities, values, power and relationships of men and women. A set of stereotypical norms prevail in most settings that associate sexual prowess with being a "man" while the same is stigmatized for girls and women. ${ }^{30,31}$ Evidence shows that agreement with such stereotypical norms has consequences for adolescent SRHR, such as masculinity norms pressuring young men to use violence and take sexual risks. ${ }^{30}$ Developing attitudes that support gender equal norms are thus an important part of healthy sexuality development.

Respect for human rights and understanding of consent highlights the fundamental connection between sexuality and health with human rights, focusing on the ability to demonstrate respect, tolerance and empathy for others irrespective of their background. ${ }^{25,26}$ Such skills involve the application of personal values and moral reasoning to distinguish between right and wrong with regards to how to treat ourselves and the people around us. Demonstrating respect for human rights is thus closely connected with ethics, and furthermore about understanding the nature and complexity of consent (e.g. what does it mean to say or give consent? $)^{1}{ }^{32}$ In the context of our model, respect for human rights might - for example - include positive attitudes towards sexual and reproductive rights, for example sexual minority rights and freedom from gender-based violence.

Critical reflection skills can be thought of as the ability to recognize and understand how different social and cultural structures and norms shape one's own feelings, behaviors and experiences. Adolescents in most cultural settings are growing up facing multiple, and often conflicting, messages and norms related to what types of (sexual) feelings or activities are acceptable, for whom, and under what circumstances. ${ }^{1}$ As noted by Bay-Cheng, ${ }^{33}$ critical reflection skills are essential to allow adolescents to take a step back, reflect and distance themselves from dominant social-sexual norms in way that is in line with their own values. This involves both the ability to reflect on messages related to one's own peer group but also to take the perspective of others (of different genders, ethnicity, sexual orientation, etc.).

Coping and stress management skills refers to the ability to manage different types of emotions and stress related to sexuality. Coping is a dynamic process to recognize, manage and adapt to different types of internal or external stressors. Such skills so can help adolescents to adapt to their environment and seek social support in line with their specific needs. ${ }^{34}$ They play an important role in learning from and translating negative experiences into new ideas and boundaries. ${ }^{28}$ Such skills include the ability to understand and manage reactions from other people related to one's own (sexual) preferences and desires, and to resist and "deconstruct" dominant social and cultural messages. ${ }^{1}$

Interpersonal relationship skills include the ability to communicate and negotiate with intimate partners, peers, family members and others that shape adolescents' lives. Such skills can include the capacity to "express affection, love and intimacy in ways that are consistent with one's values"; and tap into the ability both to seek information and assert one's preferences, as well as being able to listen to and respect others. ${ }^{1}$ Doing so involves the skill to ask for and give consent, as well as refusing and being able to accept refusal of consent (ref). ${ }^{1,32}$ Interpersonal relationship skills may thus help to both prevent unwanted interactions and sexual assault, and to promote positive, pleasurable intimate relationships. ${ }^{11}$ 


\section{Sexual agency and navigation}

Whether or not individuals are able to translate competencies into action depends on their agency to negotiate and navigate which intimate and/or solo sexual activities (if any) to engage in. In the context of this framework, sexual agency forms the link between competencies and wellbeing, and can be thought of as adolescent's ability to realize preferences and choices, express voice and influence and make decisions by drawing on resources at multiple levels. ${ }^{20}$ (ref). We view agency as being relational, with young people's personal agency being closely shaped by, and shaping, the agency of intimate partners as well other people in their social networks (e.g. peers, family members, teachers).

Navigation further refers to how young people make decisions about their sexuality in light of different circumstances, personal goals and wishes, and expectations. While a focus on individuals' freedom to choose is important from a rights-based perspective, it entails that young people are always in control and responsible (e.g. by avoiding unwanted sex), which may not be the case. In other words: making healthy choices and exercising agency related to sexuality can entail making the "wrong" choices, if these are the best available options for the individual at a certain point in time. , $28^{2}$

\section{Social-ecological context and opportunity structures}

Finally, the framework highlights the role of the social-ecological opportunity structures in shaping if, how and when adolescents are able to translate competencies into desired actions. ${ }^{20-}$

${ }^{23}$ While knowledge, skills and attitudes are important resources, these can be limited or annihilated by an individual's family, peer, community and broader societal context Blum, 2014 \#16\} For example, imagine an adolescent boy who is in love with one of his male peers. Whether or not he is able to translate his knowledge (e.g. about different types of romantic attractions), attitudes (that same-sex relationships are ok) and skills (e.g. to form relationships) to act on his feelings depends on his context, such as: whether homosexuality is legal in his country, whether social norms stigmatize rather than accept same-sex relationships, whether his family would be supportive, or whether he knows peers with similar experiences. The extent to which some or all of these opportunity structures are in place thus determines the boy's agency and his sense of sexual wellbeing.

\section{Conclusion and implications}

The importance of investing in adolescent sexual and reproductive health from a broad perspective was first recognized at the 1994 International Conference on Population and Development (ICPD), which called for "meeting the educational and service needs of adolescents to enable them to deal in a positive and responsible way with their sexuality" and highlighted young people's right to comprehensive sexuality education. ${ }^{35}$ Despite this vision, programs and research remain largely shaped by a traditional risk approach to adolescent sexuality. ${ }^{2,4,7,8}$. This includes evaluations of comprehensive sexuality education (CSE) programs which tend to focus on measuring outcomes related to two main public health indicators (teenage pregnancy and STIs), despite the increased call for measuring 'soft outcomes' (such as gender attitudes, relationships skills, etc.) as important in their own right. ${ }^{25,36}$ While the past 25 years has seen significant progress in adolescent SRHR, data and evidence on sexual wellbeing outcomes remain scant - especially in low- and middle-income countries. $^{8}$

In response to the continued push towards positive approaches, this paper presents a conceptual framework that can be used to inform both research and action. First, the framework can provide concrete direction for designing and evaluating adolescent SRHR initiatives that are rights-based and gender transformative. It can help to articulate a Theory of Change for how CSE programs, for example, can contribute to sexual wellbeing by strengthening adolescents' 
skills to consider and weigh the options and scenarios that are available to them, even if they are few. It shows that merely giving information is not enough, but that adolescents need skills to critically reflect and navigate messages, including those communicated via pornography and sexual media. ${ }^{37}$

Second, the framework can be used to guide the operationalization of measures that tap into the complex construct of sexual wellbeing from both personal and relational aspects, depending on what is most relevant and feasible. The Global Early Adolescent Study, for example, uses a number of indicators to measure young adolescents' personal sense of sexual wellbeing (e.g. body image, comfort with pubertal development, self-efficacy), as illustrated in a recent study using data from Indonesia. ${ }^{38,39}$

Third, our assets-based, life-course approach can further be used to make the case to donors and policymakers for why early investments are needed to achieve wellbeing over time. There is growing attention to consider the value of non-health outcomes in cost evaluations ${ }^{40}$ and the framework can serve as a foundation for moving beyond traditional economic measures such as quality-adjusted life years (QUALYs).

When using the framework, researchers, donors and program implementers need to consider carefully the broader experiences and context of adolescents' lives. Young people's ability to translate knowledge, skills and attitudes gained via any program into desired actions depend on the personal, social and economic resources or assets available to them, their (sexual) agency, and on the influence of institutional structures such as policies, laws and norms. This means that the specific content and framing of many of the domains in the framework will vary depending on the geographical, social, economic and cultural setting where it is being applied. More research is needed to understand sexual wellbeing and what this entails from the perspective of young people themselves and their social contexts, beyond high-income Western countries where most existing frameworks and empirical data come from today.

Nonetheless, the framework's grounding in literature on adolescent development as well as internationally agreed definitions of sexuality suggests that it is both relevant and possible to use across different contexts. In some settings it might serve as a starting point for implementers, researchers and policy makers to set priorities and make the case for viewing adolescent sexuality from a positive perspective. In others, it can help to reaffirm and operationalize what public health professionals across the globe have known for decades: that adolescent health and wellbeing, including their sexual wellbeing, is more than just the absence of disease. ${ }^{4}$ We hope that the model can serve as a roadmap to realize the ICPD vision of allowing young people to navigate their sexuality in a "positive and responsible" way. ${ }^{35}$

\section{Acknowledgements}

The authors would like to thank global experts in the field of adolescent SRHR for their input and feedback on the framework during workshops, conferences and webinars. In particular we would like to thank Rutgers colleagues Marianne Cense, Ine Vanwesenbeeck, Anna Page and Judith Westeneng; Doortje Braeken (independent consultant); the Global Early Adolescent Study (GEAS) partners, and specifically Robert Blum and Caroline Moreau at Johns Hopkins Bloomberg School of Public Health and Kristien Michielsen and Sara de Meyer at the International Center for Reproductive Health at Ghent University. Work on this paper was made possible with generous support from the Bill \& Melinda Gates Foundation [INV-008426].

\section{References}


1. Arbeit MR. What does healthy sex look like among youth? Towards a skills-based model for promoting adolescent sexuality development. Human Development. 2014;57(5):28-28.

2. Halpern CT. Reframing research on adolescent sexuality: healthy sexual development as part of the life course. Perspectives on Sexual and Reproductive Health. 2010;42(1):6-7.

3. Harden KP. A Sex-Positive Framework for Research on Adolescent Sexuality. Perspectives on Psychological Science. 2014;9(5):455-469.

4. Landers S, Kapadia F. The Public Health of Pleasure: Going Beyond Disease Prevention. American Journal of Public Health. 2020;110(2):140-141.

5. Michielsen K, De Meyer S, Ivanova O, et al. Reorienting adolescent sexual and reproductive health research: reflections from an international conference. (1742-4755 (Electronic)).

6. Russell ST. Conceptualizing positive adolescent sexuality development. Sexuality Research and Social Policy. 2005;2(3):4.

7. Tolman DL, McClelland SI. Normative Sexuality Development in Adolescence: A Decade in Review, 2000-2009. Journal of Research on Adolescence. 2011;21(1):242255.

8. Liang M, Simelane S, Fortuny Fillo G, et al. The State of Adolescent Sexual and Reproductive Health. Journal of Adolescent Health. 2019;65(6):S3-S15.

9. Blum R, Astone N, Decker M, Mouli VC. A conceptual framework for early adolescence: a platform for research. International journal of adolescent medicine and health. 2014;26(3):321-331.

10. Santelli JS, Kantor LM, Grilo SA, et al. Abstinence-Only-Until-Marriage: An Updated Review of U.S. Policies and Programs and Their Impact. Journal of Adolescent Health. 2017;61(3):273-280.

11. Lorimer K, DeAmicis L, Dalrymple J, et al. A Rapid Review of Sexual Wellbeing Definitions and Measures: Should We Now Include Sexual Wellbeing Freedom? J Sex Res. 2019;56(7):843-853.

12. Lerner RM. Developmental science, developmental systems, and contemporary theories of human development. Handbook of child psychology. 2007;1.

13. Lerner RM, Lerner JV, Almerigi JB, et al. Positive youth development, participation in community youth development programs, and community contributions of fifthgrade adolescents: Findings from the first wave of the 4-H study of positive youth development. The journal of early adolescence. 2005;25(1):17-71.

14. Patton GC, Sawyer SM, Santelli JS, et al. Our future: a Lancet commission on adolescent health and wellbeing. The Lancet. 2016;387(10036):2423-2478.

15. Anderson R. Positive sexuality and its impact on overall well-being. Bundesgesundheitsblatt-Gesundheitsforschung-Gesundheitsschutz. 2013;56(2):208214.

16. World Health Organization. Defining sexual health. https://www.who.int/teams/sexual-and-reproductive-health-and-research/key-areas-ofwork/sexual-health/defining-sexual-health. Accessed Dec 11, 2020.

17. Starrs AM, Ezeh AC, Barker G, et al. Accelerate progress-sexual and reproductive health and rights for all: report of the Guttmacher-Lancet Commission. (1474-547X (Electronic)).

18. Haffner DW. Facing facts: sexual health for America's adolescents: the report of the National Commission on Adolescent Sexual Health. SIECUS Rep. 1995;23(6):2-8. 
19. McKee A, Albury K, Dunne M, et al. Healthy sexual development: A multidisciplinary framework for research. International Journal of Sexual Health. 2010;22(1):14-19.

20. Edmeades J, Mejia C, Parsons J, Sebany M. A Conceptual Framework for Reproductive Empowerment: Empowering Individuals and Couples to Improve their Health (Background Paper). . Washington, DC: International Center for Research on Women;2018.

21. van Eerdewijk A, Wong F, Vaast C, Newton J, Tyszler M, Pennington A. White paper: A conceptual model on women and girls' empowerment. 2017.

22. Kabeer N. Resources, agency, achievements: Reflections on the measurement of women's empowerment. Development and change. 1999;30(3):435-464.

23. Kwauk C, Braga A. Translating Competencies to Empowered Action. Center for Universal Education, The Brookings Institution(November) Retrieved from https://www brookings edu/wp-content/uploads/2017/11/translating-competenciesempowered-action pdf. 2017.

24. Elder GH. Perspectives on the life course. In: Elder GH, ed. Life course dynamics: Trajectories and transitions, 1968-1980. Ithaca, NY: Cornell University Press; 1985:23-49.

25. Constantine NA, Jerman P, Berglas NF, Angulo-Olaiz F, Chou CP, Rohrbach LA. Short-term effects of a rights-based sexuality education curriculum for high-school students: a cluster-randomized trial. BMC Public Health. 2015;15(1471-2458 (Electronic)).

26. Berglas NF, Constantine NA, Ozer EJ. A Rights-Based Approach to Sexuality Education: Conceptualization, Clarification and Challenges. Perspectives on Sexual and Reproductive Health. 2014;46(2):63-72.

27. Levy JK, Darmstadt GL, Ashby C, et al. Characteristics of successful programmes targeting gender inequality and restrictive gender norms for the health and wellbeing of children, adolescents, and young adults: a systematic review. Lancet Glob Health. 2020;8(2):e225-e236.

28. Cense M. Rethinking sexual agency: Proposing a multicomponent model based on young people's life stories. Sex Education. 2019;19(3):247-262.

29. UNESCO. International technical guidance on sexuality education: an evidenceinformed approach. Revised edition. Paris: UNESCO;2018.

30. Heise L, Greene ME, Opper N, et al. Gender inequality and restrictive gender norms: framing the challenges to health. The Lancet. 2019;393(10189):2440-2454.

31. Kågesten A, Gibbs S, Blum RW, et al. Understanding Factors that Shape Gender Attitudes in Early Adolescence Globally: A Mixed-Methods Systematic Review. PloS one. 2016;11(6): $\mathrm{e} 0157805-\mathrm{e} 0157805$.

32. Fenner L. Sexual Consent as a Scientific Subject: A Literature Review. American Journal of Sexuality Education. 2017;12(4):451-471.

33. Bay-Cheng LY. Recovering empowerment: De-personalizing and re-politicizing adolescent female sexuality. Sex Roles. 2012;66(11-12):713-717.

34. Compas BE, Reeslund KL. Processes of risk and resilience during adolescence. 2009.

35. United Nations Population Fund (UNFPA). Programme of action of the International Conference on Population Development. 20th Anniversary ed. New York: UNFPA;2014.

36. UNESCO. Review of the evidence on sexuality education. Report to inform the update of the UNESCO International Technical Guidance on Sexuality Education; prepared by Paul Montgomery and Wendy Knerr, University of Oxford Centre for EvidenceBased Intervention. Paris: UNESCO;2016. 
37. Nelson KM, Rothman EF. Should Public Health Professionals Consider Pornography a Public Health Crisis? American Journal of Public Health. 2020;110(2):151-153.

38. Kågesten A, Pinandari A, Page A, Wilopo SA, van Reeuwijk M. Sexual wellbeing among young adolescent boys and girls in Indonesia In. Submitted to American Journal of Public health (under review) 2021.

39. UGM Center for Reproductive Health. Early Adolescent's Health in Indonesia: Evidence Base from GEAS-Indonesia: Baseline 2019. Yogyakarta: UGM;2019.

40. Benning TM, Alayli-Goebbels AFG, Aarts M-J, et al. Exploring Outcomes to Consider in Economic Evaluations of Health Promotion Programs: What Broader Non-Health Outcomes Matter Most? BMC Health Services Research. 2015;15(1):266. 